http://jmscr.igmpublication.org/home/ ISSN (e)-2347-176x ISSN (p) 2455-0450 crossref DOI: https://dx.doi.org/10.18535/jmscr/v8i12.03

\author{
Journal Of Medical Science And Clinical Research

\title{
Comparative evaluation of compressive strength of different packable composites with different cavity configurations - An in Vitro Study
}

\author{
Authors \\ Humanaz Shaikh $^{1 *}$, Sameer Jadhav ${ }^{2}$, Vivek Hegde $^{3}$, Mohammad Naved $^{4}$ \\ ${ }^{1}$ Postgraduate student, Department of Conservative Dentistry and Endodontics, M.A. Rangoonwala College of Dental \\ Sciences and Research Centre Pune \\ ${ }^{2}$ Professor, Department of Conservative Dentistry and Endodontics, M.A. Rangoonwala College of Dental Sciences \\ and Research Centre Pune \\ ${ }^{3}$ Head of the department, Department of Conservative Dentistry and Endodontics, M.A. Rangoonwala College of \\ Dental Sciences and Research Centre Pune \\ ${ }^{4}$ Postgraduate student, Department of Conservative Dentistry and Endodontics, M.A. Rangoonwala College of Dental \\ Sciences and Research Centre Pune \\ *Corresponding Author \\ Humanaz Shaikh
}

\begin{abstract}
Background: The application of excessive force on a material results in fracture of material which may be complete or incomplete. Resistance to fracture is an important property of a material. It would depend on its composition and also on the cavity configuration in which it is being placed. The cavity configuration plays an important role in the durability of the restored material. The preparation also maximizes or minimizes the bondable enamel surface area. Restoring the tooth with appropriate material is also an important aspect as its long-term performance depends on the physical and mechanical properties. It is determined by its compressive properties and the composition.

Short running title: Effect of cavity preparations on the strength of different composites

Aim: To evaluate the effect of the different cavity preparations on the strength of different composites as a restorative material.

Methodology: Clark's Class II and Class II box only cavities were prepared on the distal surface of all mandibular molars of permanent dentition and were restored with two different packable composite, Filtek Z350 (3M/ESPE, Maplewood, USA), a nanofilled type of composite \& Tetric N-Ceram (Ivoclar Vivadent, Schaan, Liechtenstein), a nanohybrid type. Compressive strengths of both cavities and materials were measured using the computer control universal testing machine. The data was statistically analysed using one way anova.

Results: Results were subjected to statistical analysis one-way analysis of variance test and Student's Unpaired ttest. The compressive strength was highest for Clarks Class II cavity preparation as compared to the conventional class II box only cavity preparation. The Tetric N-Ceram (Ivoclar Vivadent) showed the highest compressive strength than FiltekZ350 (3M/ESPE)

Conclusion: Within the limitations of the study, it can be concluded Clarks Class II cavity preparation and Tetric $N$ Ceram (Ivoclar Vivadent) has highest compressive strength.

Clinical Significance: Cavity preparation in which the margins of the proximal preparations extend into enamel and make the tooth more resistant to fracture and also increasing durability of restoration. Dental composites cause Polymerization shrinkage leads to micro leakage and thus creating gaps at the dentin or restoration interface and increase chances of fracture.

Keywords: Clark's class II cavity, Class II box only, composites, compressive strength, fracture resistance, nanofilled composite.
\end{abstract}




\section{Introduction}

A successful restoration that closely resembles a natural tooth can be provided by proper technique and insertion of composite. Posterior composites usage has increased with time due to improvements in the physical properties of materials and better adhesion. ${ }^{1}$ Such cavity preparations cause reduction in the tooth strength due to loss of marginal ridges and micro cracks because of repeated occlusal loading. In conventional class II cavities, loads applied occlusally may lead to fracture of the cusps. The fracture of the restration under occlusal compressive loads depend upon the type of the composite resin used. $^{2}$

Preservation of sound tooth structure during cavity preparation is an advantage of restoring the posterior teeth with composite. ${ }^{3}$ Bulk of strength is provided by the sound tooth structure. ${ }^{4}$ Thus the cavity configuration is an important aspect as per providing the strength to the tooth as well as the restoration placed in the cavity. The preparations which aids in conserving tooth structure are the box-only, slot, tunnel preparations. ${ }^{5}$

In class II the stresses which tend to rotate the restoration mostly act on marginal ridges. The forces acting on marginal ridges has two components horizontal and vertical in which the horizontal components of the two teeth counteract each other and vertical are resolved normally by the underlying tissues.

The box only class II cavity preparation has sharp internal line angles thus cause initiation of crack and vulnerable dentin gets more prone to fracture. Therefore, to overcome this a new modified form of cavity preparation was introduced by David Clark in which the margins of the proximal preparations extend into enamel and making the tooth more resistant to fracture and also increasing durability of restoration. ${ }^{7}$ Clark promotes cavity designs that are based on the adhesion of restorative materials.

Dental composites having near ideal mechanical properties, ease of handling and good adhesion with enamel and dentin leads to increase in the longevity of the restoration. Few are recommended in load bearing areas and few in aesthetic areas. ${ }^{6}$ Polymerization shrinkage leads to micro leakage and thus creating gaps at the dentin or restoration interface and increase chances of fracture. $^{6}$

The aim of this study was to evaluate the effect of the different cavity preparations on the compressive strength of different composites as a restorative material.

\section{Materials and Methods}

A) Sample Collection: After obtaining clearance from the Institutional Ethical Committee, forty human mandibular molars of permanent dentition extracted because of compromised periodontium. Teeth free of apical resorption and caries were used for the study. Informed consent of the patient was obtained before collecting their teeth.

Inclusion Criteria: Non-carious mandibular molars with well-formed apices.

Exclusion criteria: Grossly carious teeth, teeth with development anomalies, teeth with resorption.

The collected teeth were stored in $10 \%$ formalin (Microtonics, India) throughout the study. The teeth were debrided ultrasonically and were then mounted in resin acrylic blocks such that the cemento-enamel junction was exposed.

\section{B) Sample Distribution}

The teeth were divided into two groups $(\mathrm{N}=20)$

Group A: Clark's Class II preparation (Figure 1) Group A was divided into two subgroups bases on two different packable composite used for restoration;

Subgroup 1: Filtek Z350 a nanofilled type of composite (3M/ESPE, Maplewood, USA) $(n=10)$

Subgroup 2: Tetric N-Ceram $(n=10)$ nanohybrid type (Ivoclar Vivadent, Schaan, Liechtenstein) 
Group B: Class II box only preparation (Figure 2) Group B was divided into two subgroups bases on two different packable composite used for restoration;

Subgroup 1: Filtek Z350 a nanofilled type of composite (3M/ESPE, Maplewood, USA) $(\mathrm{n}=10)$

Subgroup 2: Tetric N-Ceram $(\mathrm{n}=10)$ nanohybrid type (Ivoclar Vivadent, Schaan, Liechtenstein)

Clark's class II cavity design is a saucer shape with serpentine/disappearing margins.

Class II cavities with Clarks configuration were prepared on the distal surfaces of 20 molars with standard cavity measurements using a Fissurotomy Bur System (SS White, Lakewood, NJ, USA)

C) Cavity Preparation: Class II cavities were prepared on the distal surface of all the teeth. High speed air rotor was used. All cavities had a standard bucco-lingual width of $2 \mathrm{~mm}$, mesio-distal width of $1 \mathrm{~mm}$, occluso-gingival height of $2.5 \mathrm{~mm}$ and axial depth of $1 \mathrm{~mm}$.

\section{D) Sample restorations}

Samples in subgroups A1 and B1 were restored with Filtek Z350 a nanofilled composite.

Samples in subgroups A2 and B2 were restored with Tetric N-Ceram $(n=10)$ nanohybrid composite.

A saddle contoured metal matrix (Filay Dent) was used. Cavities were etched with $37 \%$ phosphoric acid then water rinsed followed by application of adhesive (Tetric* N-Bond). Packable composite resin was placed in oblique layers in increments and cured for 20 seconds using a blue phase curing light of $1200 \mathrm{~mW} / \mathrm{cm} 2$.All the composite restorations were finished and polished with a composite polishing kit, Super Snap (Shofu).

\section{E) Evaluation of compressive strength}

The samples were placed in the Universal testing machine for mechanical testing
(ACME Engineers, India) (Figure 3). Each block was subjected to compressive load with the tip placed on restored cavity portion along the long axis of the tooth at crosshead speed of $3 \mathrm{~mm} / \mathrm{min}$ until restoration fractured and values were recorded.

\section{Statistical Analysis}

The data was compared for differences using oneway analysis of variance, followed by multiple comparisons using Bonferroni's Post-Hoc test. Probability, $\mathrm{P}<0.05$ : considered as statistically significant.

\section{Results}

Comparison between different cavity preparation The compressive strength was highest for Clarks Class II cavity preparation (Group A) as compared to the conventional class II box only cavity preparation (Group B). The results were statistically significant (Table $1 \&$ graph 1 ).

Comparison between materials

The Tetric N-Ceram (Ivoclar Vivadent) showed the highest compressive strength than FiltekZ350 (3M/ESPE). (Table $1 \&$ graph 1 )

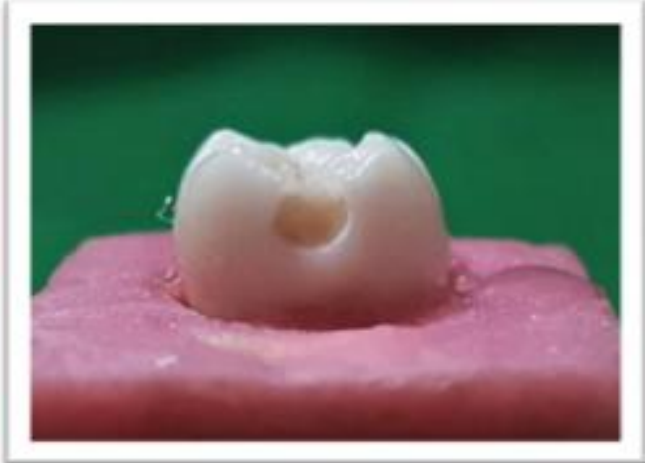

Figure 1: Clark's Class II cavity preparation (Group A)

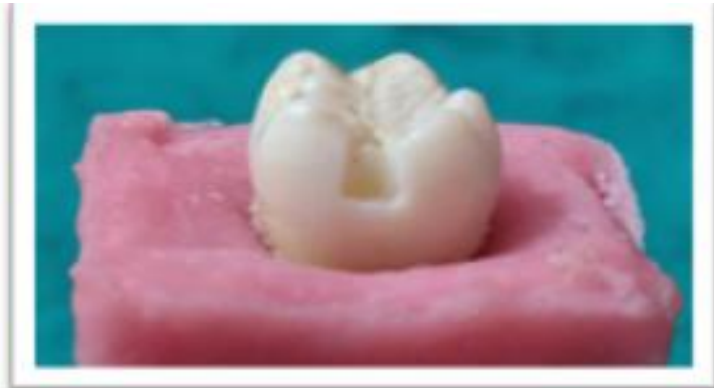

Figure 2: Class II box only preparation (Group B) 


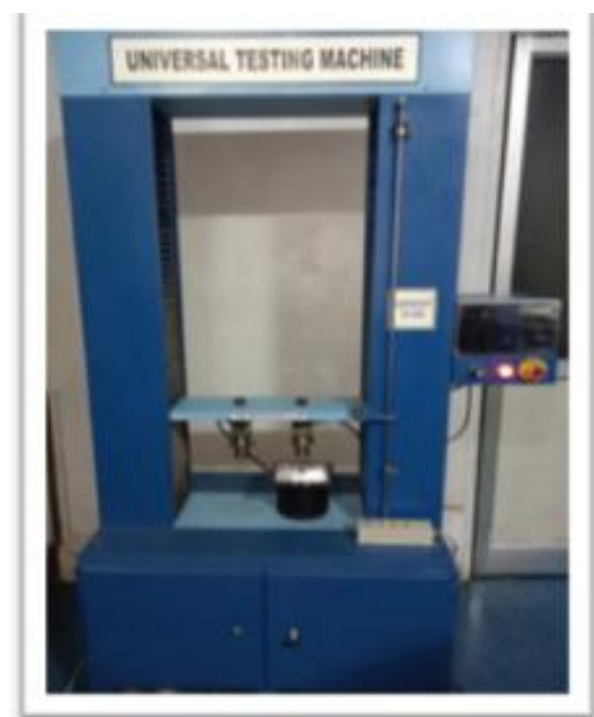

Figure 3: Universal testing machine

Table 1) Intragroup distribution of mean compressive strength (MPa)

\begin{tabular}{|l|c|c|c|}
\hline Groups & Subgroups & Compressive strength (Mean \pm SD) & P-value \\
\hline Group A & Filtek Z350 & 402.4 & $<0.05$ \\
\cline { 2 - 3 } Clark`s Class II & Tetric N-Ceram & 456.2 & \\
\hline Group B & Filtek Z350 & 250.1 & $<0.05$ \\
\cline { 2 - 4 } Class II box only & Tetric N-Ceram & 326.4 & \\
\hline
\end{tabular}

Graph 1: Intergroup distribution of mean compressive strength

\section{Mean compressive strength ( $\mathrm{MPa}$ )}

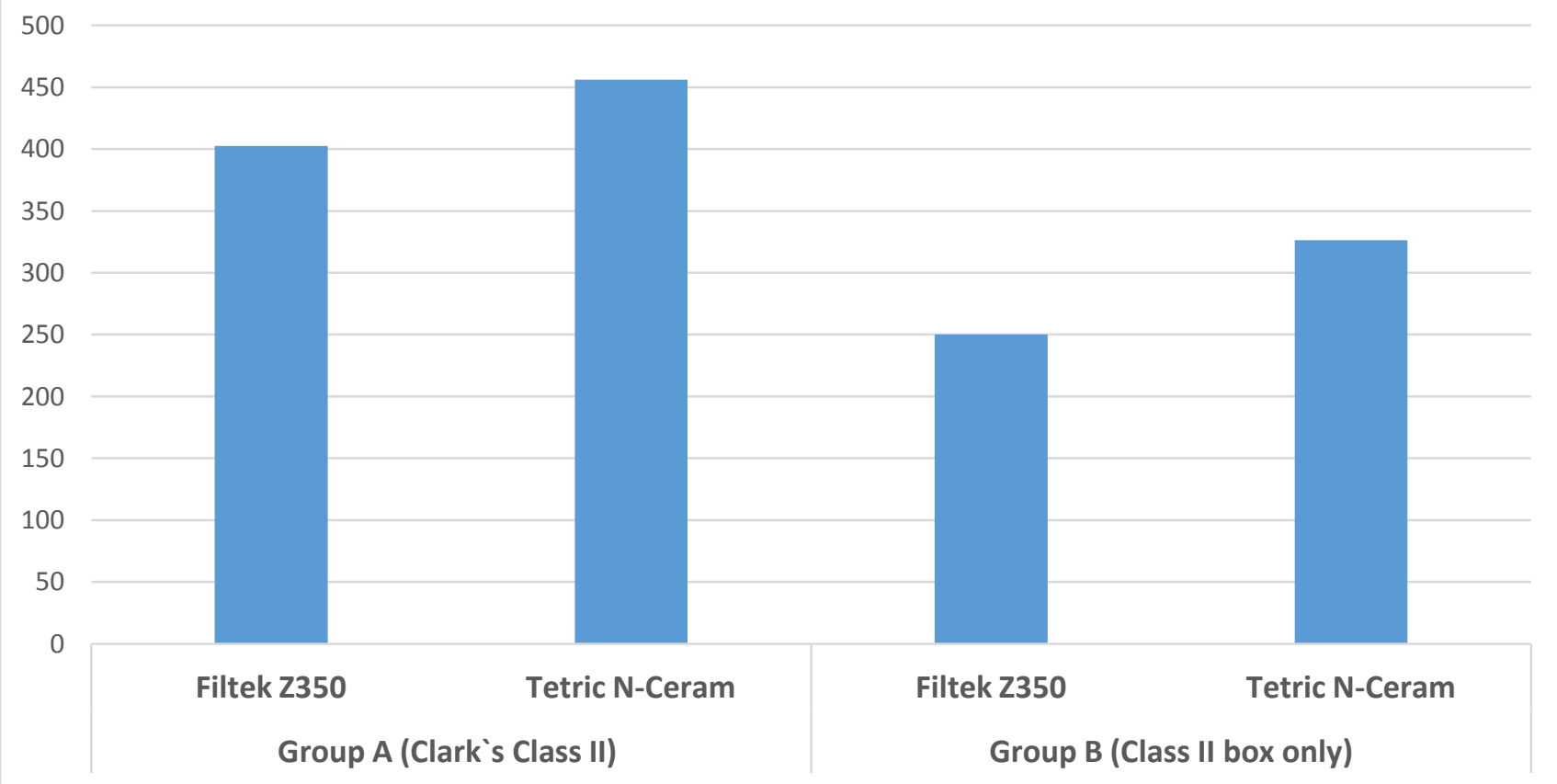

\section{Discussion}

The composite resins now a days preferred restorative material for restoration of teeth because they are adhesive in nature. Composite resins don't require conventional cavity for retention. The composite restorations in posterior 
teeth are critical because of higher masticatory forces.

The application of excessive force on a material results in fracture of material which may be complete or incomplete. Resistance to fracture is an important property of a material. It would depend on its composition and also on the cavity configuration in which it is being placed. ${ }^{9} \mathrm{An}$ increase and decrease in fracture resistance depend on the stress applied on it. Clinically, masticatory forces are applied constantly on the restored or unrestored teeth. The design of a cavity can cause reduction in these stresses and thus may have a significant effect on the fracture resistance of material and tooth ${ }^{1-9}$.

The cavity designs used in this study were Class II box only and Clarks Class II cavity preparation. The Clarks class II is a modification to classic cavity preparation to class II preparation given by G.V.Black ${ }^{7}$ Clark's class II cavity is as such a slot preparation and also more conservative than the Class II box shaped cavity. ${ }^{1}$ The conservative micro preparations can be done ideally with the Fissurotomy Bur (SS White, Lakewood, NJ, USA). ${ }^{7}$

The cavity configuration plays an important role in the durability of the restored material. The result of this study is consistent with study done by Kumar $\mathbf{T}$ et al. ${ }^{\mathbf{1}}$ In this study samples in Class II box preparation demonstrated lesser compressive strength as compared to Clarks preparation. The reasons might be that the Class II box shape preparation has sharp internal line angles thus can cause crack initiation and make the material or tooth more prone to fracture. ${ }^{10}$ Presence of more enamel margin provides better bonding. A stronger ionic bond can be formed by the calcium present in hydroxyapatite crystals. Thus, Clarks cavities provide better bonding due to more enamel surface as the cavities are saucer/slot shaped with serpentine/disappearing margins. ${ }^{8}$

Minimally Invasive dentistry is an upgrade of Minimally Traumatic dentistry. ${ }^{7}$ It is the maximum preservation of healthy dental structures. ${ }^{11}$ The goal of minimal invasive technique is to extend the lifetime of restored teeth with minimal intervention. $^{12}$

Restoring the tooth with appropriate material is also an important aspect as its long-term performance depends on the physical and mechanical properties. It is determined by its compressive properties and the composition. ${ }^{6}$

The composite material used in this study were those with the nanosized filler particles Filtek Z350 and Tetric N-Ceram. The compressive strength varies for every composite according to the filler particle size. ${ }^{6}$ Thus, there was a significant difference between compressive strength of Filtek Z350 and Tetric N-Ceram.

\section{Conclusion}

Within the limitations of the study, it can be concluded that Clarks Class II cavity restored with Tetric N-Ceram-nanohybrid composite (Ivoclar Vivadent) has highest compressive strength. Clarks Class II cavity design can be used for the small carious lesion in proximal surfaces of posterior teeth.

\section{References}

1. Kumar T, Sanap A, Bhargava K, Aggarwal S, Kaur G, Kunjir K. Comparative evaluation of the bond strength of posterior composite with different cavity configurations and different liners using a two-step etch and rinse adhesive system: In vitro study. J Conserv Dent 2017;20:166-9

2. Hamoudaa IM, Shehatab SH. Fracture resistance of posterior teeth restored with modern restorative materials. J Biomed Research 2011,25(6):418-424

3. Leinfelder KF. A conservative approach to placing posterior composite resin restorations. J Am Dent Assoc 1996;127: 743- 8

4. Sano H. Microtensile testing, nanoleakage, and biodegradation of resin- dentin bonds. J Dent Res 2006;85:11- 4 
5. Castillo MD. Class II composite marginal ridge failure: Conventional vs. proximal box only preparation. J Clin Pediatr Dent 1999;23:131- 6

6. Abuelenain DA, Neel EAA and Al-Dharrab A. Surface and Mechanical Properties of Different Dental Composites. Austin J Dent. 2015;2(2): 1019

7. Clark D. Introducing the Clark Class I and II restoration. Oral Health 2009. p. 82- 91

8. Yoshida Y, Nagakane K, Fukuda R, Nakayama Y, Okazaki M, Shintani H, et al. Comparative study on adhesive performance of functional monomers. J Dent Res 2004;83:454- 8

9. Moosavi $\mathrm{H}$, Zeynali $\mathrm{M}$ and Pour $\mathrm{ZH}$. Fracture Resistance of Premolars Restored by Various Types and Placement Techniques of Resin Composites. Int $\mathbf{J}$ of Dent.2012,ArticleID 973641,5pages

10. Albers HF. Tooth Coloured Restorations. 8th ed. Santa Rosa: Alta Books; 1997

11. Ericson D, Kidd E, McComb D, Mjor I, Noack MJ.Minimal Invasive Dentistryconcepts and techniques in cariology. Oral Health Prev Dent.2003;1(1):59-72

12. Peters MC, McLean ME. Minimally invasive operative care.I. Minimal intervention and concepts for minimally invasivecavity preparations. $\mathbf{J}$ Adhes Dent.2001 Spring;3(1):7-16. 